\title{
Exon array analysis reveals neuroblastoma tumors have distinct alternative splicing patterns according to stage and MYCN amplification status
}

\author{
Xiang Guo ${ }^{1,2+}$, Qing-Rong Chen ${ }^{1+}$, Young K Song ${ }^{1}$, Jun S Wei ${ }^{1}$ and Javed Khan ${ }^{1 *}$
}

\begin{abstract}
Background: Neuroblastoma (NB) tumors are well known for their pronounced clinical and molecular heterogeneity. The global gene expression and DNA copy number alterations have been shown to have profound differences in tumors of low or high stage and those with or without MYCN amplification. RNA splicing is an important regulatory mechanism of gene expression, and differential RNA splicing may be associated with the clinical behavior of a tumor.
\end{abstract}

Methods: In this study, we used exon array profiling to investigate global alternative splicing pattern of 47 neuroblastoma samples in stage 1 and stage 4 with normal or amplified MYCN copy number (stage 1-, 4- and 4+). The ratio of exon-level expression to gene-level expression was used to detect alternative splicing events, while the gene-level expression was applied to characterize whole gene expression change.

Results: Principal component analysis (PCA) demonstrated distinct splicing pattern in three groups of samples. Pairwise comparison identified genes with splicing changes and/or whole gene expression changes in high stage tumors. In stage 4- compared with stage 1- tumors, alternatively spliced candidate genes had little overlap with genes showing whole gene expression changes, and most of them were involved in different biological processes. In contrast, a larger number of genes exhibited either exon-level splicing, gene-level expression or both changes in stage 4+ versus stage 1-tumors. Those biological processes involved in stage 4- tumors were disrupted to a greater extent by both splicing and transcription regulations in stage 4+ tumors.

Conclusions: Our results demonstrated a significant role of alternative splicing in high stage neuroblastoma, and suggested a MYCN-associated splicing regulation pathway in stage 4+ tumors. The identification of differentially spliced genes and pathways in neuroblastoma tumors of different stages and molecular subtypes may be important to the understanding of cancer biology and the discovery of diagnostic markers or therapeutic targets in neuroblastoma.

\section{Background}

Alternative splicing of pre-messenger RNA is nearly universal, involving more than $90 \%$ of human genes [1]. It is an important regulatory mechanism of gene expression for tissue-specific functions. Each gene maintains a delicate balance of its alternative transcripts in normal cells, disruption of which affects normal cellular processes and may cause various diseases, including cancer

\footnotetext{
* Correspondence: khanjav@mail.nih.gov

+ Contributed equally

'Oncogenomics Section, Pediatric Oncology Branch, National Cancer Institute, National Institute of Health, Gaithersburg, MD 20877, USA Full list of author information is available at the end of the article
}

[2]. Although there have been numerous studies to identify tumor-specific splicing variants as diagnostic markers or therapeutic targets, only recently has alternative splicing in cancer been studied using genome-wide profiling methods [3-7]. Most of these studies focus on the identification of splicing variants in tumor, while little has been explored on the role of alternative splicing in tumors of different stages and molecular subtypes.

Neuroblastoma is the most common solid extracranial tumor in children. The incidence rate is 10.2 cases per million children under 15 years of age, and the median age at diagnosis is 17 months [8]. Stage, age, and other biological features in tumour cells are important 
prognostic factors for risk stratification and disease management. The "International Neuroblastoma Staging System" (INSS) classifies the tumor into six stages (1, $2 \mathrm{~A}, 2 \mathrm{~B}, 3,4,4 \mathrm{~S})$ according to its anatomical presence at diagnosis [9]. Localized disease has favorable outcome with an overall survival rate for stage 1 disease of 75$90 \%$. Patients over 18 months with stage 4 disease has a 2-year disease-free survival rate of only 30-40\% [10]. $M Y C N$ is the most important biologic marker for neuroblastoma. It is amplified in approximately $25 \%$ of de novo neuroblastoma cases and is more common in patients with advanced-stage disease. $M Y C N$-amplified tumor is highly aggressive with poor outcome [11]. Alternative splicing has been shown to be involved in neuroblastoma development [12]. For example, kinesin family member $1 \mathrm{~B}$ isoform beta (KIF1Bbeta) but not alpha is down-regulated in advanced stages of neuroblastoma. KIF1Bbeta induces apoptotic cell death, suggesting its role as a haploinsufficient tumor suppressor $[13,14]$. In this study, we used Affymetrix Human Exon 1.0 ST Array (HuEx) to measure exon expression levels in 47 neuroblastoma samples of different clinical stages and molecular subtypes including stage 1 with normal $M Y C N$ copy number (1-), stage 4 with $M Y C N$ amplification (4+) or normal $M Y C N$ copy number (4-). The goal was to identify stage- and $M Y C N$ amplification-specific splicing patterns in comparison to whole gene expression changes for the understanding of cancer biology and discovery of biomarkers or therapeutic targets in neuroblastoma.

\section{Results}

Identification of alternatively spliced candidate genes

To study the role of splicing regulation in high stage and $M Y C N$ amplified neuroblastoma, we used $\mathrm{HuEx}$ array to measure exon expression levels in 47 neuroblastoma samples from 10 stage 1-, 28 stage 4-, and 9 stage $4+$ tumors (Table 1 ). HuEx array allows the detection of differential inclusion or skipping of exons by measuring expression levels of individual exons in different groups of samples. Alternative splicing events may be detected by normalized intensity (NI), which is defined as the ratio of exon-level probeset expression to gene-level transcript cluster expression [15]. To examine the global splicing pattern in neuroblastoma, we performed principal component analysis (PCA) using NI values of all core probesets $(n=221,809)$ across all samples after quality filtering. Figure 1 shows that stage 1 - and 4+ samples are clearly separated from each other, while stage 4- samples are located between the other two groups. The separation of three groups of tumors by NI values suggests distinct alternative splicing patterns associated with clinical stage and $M Y C N$ status.
Probesets with significantly different NI values in two groups of samples represent exons that may be differentially spliced in two disease states. We used Significant Analysis of Microarray (SAM) procedure [16] with a stringent false discovery rate threshold ( $q$ value $<0.05)$ to compare NI among three groups of patients. There were 1501 differentially spliced candidate genes between 4- and 4+, while only 362 genes were differentially spliced between 1 - and 4-. Using AltAnalyze, we checked prior evidence of alternative splicing in Ensembl and/or UCSC genome browser databases [17]. Of the candidate gene lists derived from the comparison of $4-/ 4+$ and 1-/4- tumors, $44.7 \%$ (671) of the former list and 29.6\% (107) of the latter were supported by prior evidence of alternative splicing. The largest difference was between $4+$ and 1 with 2775 differentially spliced candidate genes, of which $46.9 \%$ (1302) had alternative exons with prior evidence of alternative splicing (Additional file 1, Figure 2A). Therefore, both stage and $M Y C N$ amplification seem to affect the alternative splicing patterns in neuroblastoma.

\section{Potential impact of alternative splicing in neuroblastoma}

To estimate the functional impact of alternative splicing, we predicted changes in domain, motif, and miRNA binding site composition of protein sequences for alternatively regulated exons in stage 1-, 4-, and 4+ tumors [17]. The percentage of alternative exons that were associated with predicted domain/motif change ranges from $74.1 \%$ to $76.2 \%$ for the pair-wise comparison of three tumor groups. The number of genes including at least one alternative domain/motif were 296 (81.8\%) for stage 4- vs 1-, 1181 (78.7\%) for stage 4+ vs 4-, and 2203 (79.4\%) for stage $4+$ vs 1 - respectively. In addition to the impact on protein domain/motif, alternative splicing may result in gain or loss of miRNA binding sites. Our analysis identified 19, 280, and 538 genes containing alternative exons overlapping with predicted miRNA binding sites for the comparison of $4-/ 1-, 4+/ 4-$, and 4 $+/ 1$ - tumors, respectively. These results suggested diverse changes in protein function and expression regulated by alternative splicing in high stage neuroblastoma.

Several known aberrant splicing events in neuroblastoma and/or other tumors are evident in our candidate lists. For example, exon array data indicated decreased expression of KIF1Bbeta but not KIF1Balpha in high stage neuroblastoma, which is consistent with previous report (Additional file 2) [18].

More interestingly, our results showed increased expression of M2 isoform of PKM2 in high stage neuroblastoma while the M1 isoform exhibited decreased expression (Figure 3). The splicing switch of pyruvate kinase has been demonstrated in multiple tumor types 
Table 1 Tumor samples used in the study

\begin{tabular}{|c|c|c|c|c|c|c|}
\hline & Year of Diagnosis & Age at Diagnosis (years) & INSS Stage & MYCN Amplification Status & Clinical Outcome & Years of Survival \\
\hline NB7 & 1998 & 1.3 & 1 & NA & A & 5.2 \\
\hline NB8 & 1998 & 4.6 & 4 & NA & $\mathrm{D}$ & 1.8 \\
\hline NB9 & 1996 & 1.1 & 1 & NA & A & 7.1 \\
\hline NB17 & 2000 & 1.2 & 1 & NA & A & 3.5 \\
\hline NB21 & 2000 & 5.2 & 4 & AMP & $\mathrm{D}$ & 0.6 \\
\hline NB24 & 2000 & 0.6 & 4 & NA & A & 3 \\
\hline NB27 & 2000 & 10.5 & 4 & AMP & D & 1.4 \\
\hline NB29 & 1998 & 0.3 & 1 & NA & A & 5.1 \\
\hline NB30 & 1997 & 0.9 & 4 & NA & $A$ & 5.9 \\
\hline NB31 & 1998 & 1.4 & 4 & NA & A & 6.7 \\
\hline NB32 & 1998 & 1.2 & 4 & NA & A & 5.7 \\
\hline NB33 & 1998 & 1.4 & 1 & NA & A & 4.8 \\
\hline NB34 & 1997 & 1.2 & 1 & NA & A & 5.2 \\
\hline NB205 & 1995 & 3.9 & 4 & NA & D & 2.3 \\
\hline NB206 & 1995 & 2.7 & 4 & NA & $D$ & 5.8 \\
\hline NB207 & 1995 & 4.4 & 4 & NA & D & 3.1 \\
\hline NB208 & 1995 & 0.8 & 1 & NA & A & 4.8 \\
\hline NB210 & 1996 & 2.5 & 4 & NA & $D$ & 1.1 \\
\hline NB221 & 1997 & 0.4 & 1 & NA & A & 5.7 \\
\hline NB237 & 1999 & 4.0 & 1 & NA & A & 3.2 \\
\hline NB238 & 1999 & 1.1 & 1 & NA & A & 3 \\
\hline NB265 & 1996 & 1.8 & 4 & AMP & D & 2 \\
\hline NB266 & 1996 & 2.0 & 4 & AMP & D & 0 \\
\hline NB269 & 1997 & 0.8 & 4 & NA & A & 5.3 \\
\hline NB275 & 1995 & 1.6 & 4 & NA & $D$ & 1 \\
\hline NB278 & 1999 & 1.7 & 4 & AMP & D & 0.8 \\
\hline NB282 & 1999 & 4.6 & 4 & NA & A & 3.3 \\
\hline NB283 & 1999 & 5.5 & 4 & NA & D & 4 \\
\hline NB500 & 1995 & 5.1 & 4 & NA & A & 7.4 \\
\hline NB503 & 2000 & 3.9 & 4 & NA & A & 3.3 \\
\hline NB504 & 2000 & 0.3 & 4 & NA & A & 3.5 \\
\hline NB506 & 2000 & 0.3 & 4 & NA & $A$ & 3.9 \\
\hline NB508 & 2000 & 0.2 & 4 & NA & A & 3.2 \\
\hline NB509 & 2000 & 3.0 & 4 & NA & D & 4.1 \\
\hline NB510 & 2000 & 0.6 & 4 & NA & A & 3.4 \\
\hline NB511 & 2001 & 0.1 & 4 & NA & A & 3.0 \\
\hline NB514 & 2001 & 11.6 & 4 & NA & A & 3.5 \\
\hline NB515 & 2001 & 3.9 & 4 & NA & A & 3.2 \\
\hline NB521 & 1999 & 1.5 & 4 & NA & A & 5.6 \\
\hline NB536 & 1998 & 3.1 & 4 & NA & D & 1.2 \\
\hline NB540 & 1999 & 3.4 & 4 & NA & D & 1.1 \\
\hline NB541 & 1999 & 9.6 & 4 & AMP & D & 0.1 \\
\hline NB543 & 1999 & 11.4 & 4 & NA & $D$ & 1.4 \\
\hline NB545 & 1999 & 3.4 & 4 & AMP & D & 2.2 \\
\hline NB547 & 2000 & 1.5 & 4 & AMP & D & 3.8 \\
\hline NB548 & 2000 & 1.6 & 4 & NA & $D$ & 3.4 \\
\hline NB581 & 2002 & 1.6 & 4 & AMP & D & 1.6 \\
\hline
\end{tabular}

Abbreviations: INSS, International Neuroblastoma Staging System; AMP, amplification; NA, not amplified; A, alive without event; D, deceased due to neuroblastoma disease. 


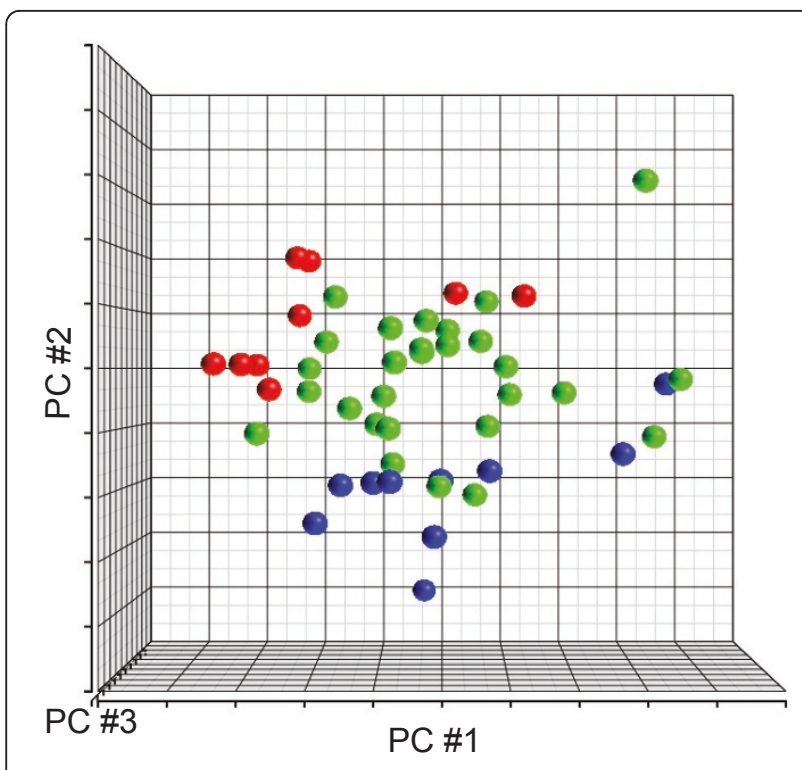

Figure 1 Principal component analysis (PCA) of 47 neuroblastoma samples by log2-transformed normalized intensity of core probesets. PCA was performed using $\mathrm{NI}$ values of core probesets $(n=221,809)$ across all samples after quality filtering. Stage 1- and 4+ samples are clearly separated from each other, while stage 4- samples are located between them. Blue, stage 1-; green, stage 4-; red, stage 4+ tumors.

[19], but not previously reported in neuroblastoma. Two isoforms are expressed through exchange of two cassette exons, which are equally long and share $60 \%$ protein sequence identity [20]. Functional annotation by AltAnalyze identified the gain of the allosteric activator fructose 1,6-bisphosphate (FBP) binding region and intersubunit contact in high stage neuroblastoma, consistent with the allosteric regulation of M2 isoform but not M1 isoform by FBP [20]. The upregulation of M2 isoform in stage $4+$ vs 1 - tumors was also evident at the proteome level demonstrated by a previous proteomics study in our lab, which applied isotope-coded affinity tags (ICAT) in combination with mass spectrometry to quantify peptide expression levels in stage 1- and 4+ tumors [21]. The quantitative ICAT analysis identified five peptides matched against PKM2 protein sequence, among which KCCSGAIIVLTKS was from the exon specific to the M2 isoform and the other four were from constitutive exons in PKM2. M2 isoform-specific peptide demonstrated a mean $\log 2$-transformed expression ratio of 2.73, and the other peptides had a mean ratio of 1.67 between $4+$ and 1 - tumors ([21]). Although no peptide has been found for M1 isoform-specific exon, the higher expression change of M2 isoform-specific exon than constitutive exons suggested the upregulation of M2 isoform but not the M1 isoform at the proteome level.

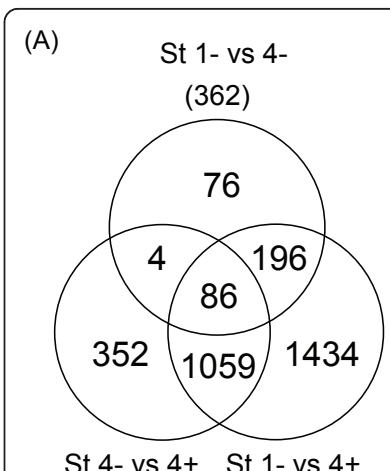

St 4- vs 4+ St 1- vs 4+

(1501)

(2775)

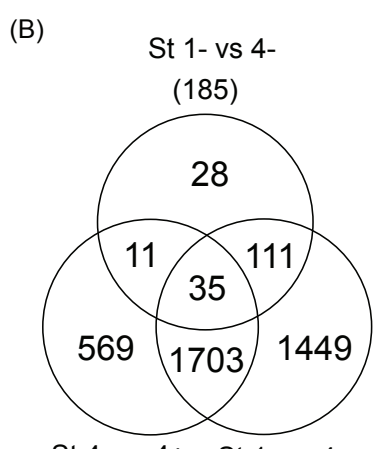

St 4- vs 4+ St 1- vs 4+

(2318)

(3298)

(C)

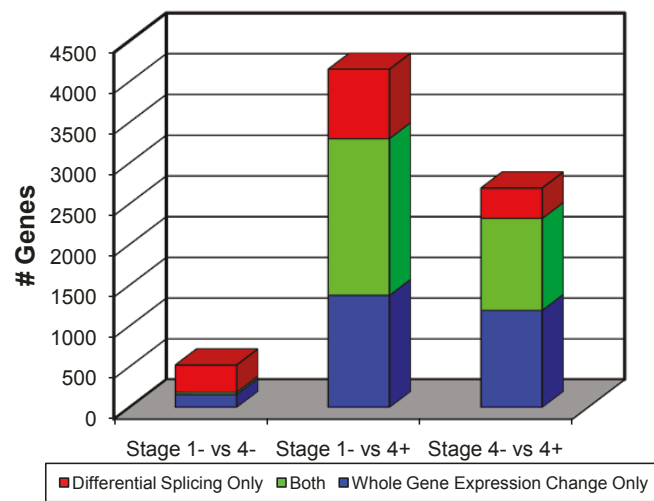

Figure 2 Comparison of exon level splicing change and gene level expression change. Pairwise comparison of stage 1-, 4-, and $4+$ tumors was performed for both exon level splicing change and gene level expression change; the genes with FDR $<0.05$ were identified. (A) Venn diagram of alternatively spliced candidate genes. (B) Venn diagram of differentially expressed candidate genes. (C) Comparison of exon level splicing change and gene level expression change. Blue column shows number of genes with whole gene expression change but not splicing change, while red column shows number of genes with splicing change but not whole gene expression change. Green column represents genes with both whole gene expression and splicing changes.

\section{Alternative splicing and whole gene expression changes in neuroblastoma}

To compare global splicing and transcription regulation in high stage neuroblastoma, we derived whole gene expression signatures by pairwise comparison of genelevel transcript cluster signals. Therefore, splicing signature includes genes having differentially included/ excluded exons between two tumor groups, while whole gene expression signature are genes with different genelevel signals of two tumor groups. SAM analysis resulted in 185,2318 , and 3298 genes showing whole gene expression changes for stage 1- vs 4-, 4- vs 4+, and 1vs $4+$, respectively (FDR $\leq 0.05$ and Fold Change $\geq 1.5$; Figure 2B, Additional file 3). 


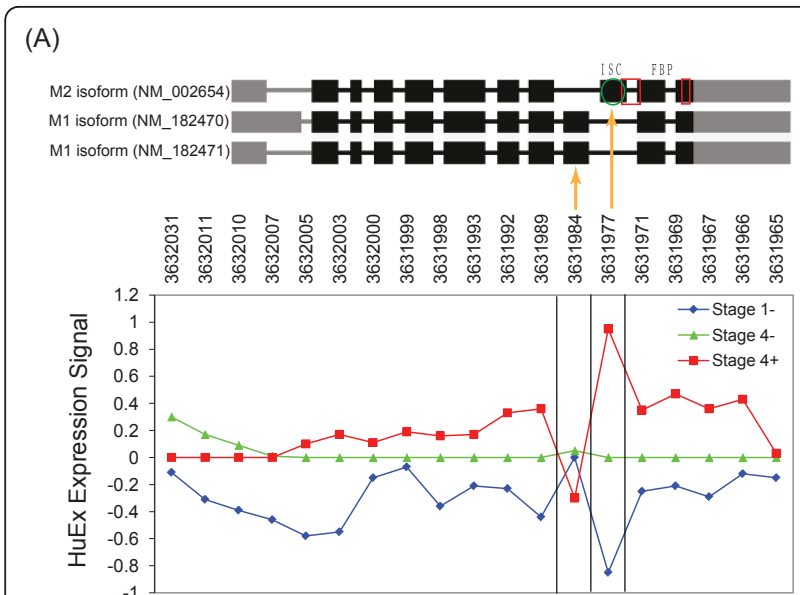

(B)

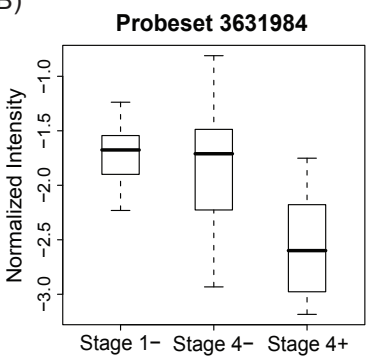

(C)

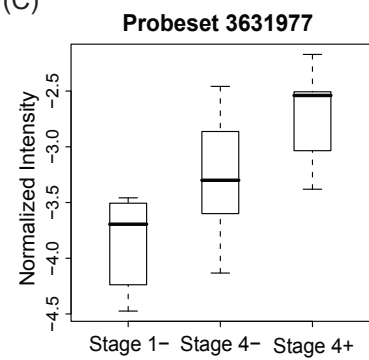

Figure 3 Alternative splicing of pyruvate kinase (PKM2) detected by Affymetrix exon (HuEx) array. (A) Gene structure of known isoforms is shown on the top panel with predicted domains/motifs that differ in protein isoforms. Green oval shows intersubunit contact (ISC) sequence, and red boxes point to fructose 1,6-bisphosphate (FBP) binding regions as defined by UniProt. The HuEx expression is shown on the bottom panel. Each point represents mean log2-expression of each group that was then median-centered across three groups. Orange lines point to probeset 3631984 that mapped to the unique exon in isoform M1 (NM_182470 and NM_182471), and probeset 3631977 that mapped to the unique exon in isoform M2 (NM_002654). While isoform M2 specific probeset showed increased expression in stage $4+$ compared to stage 4-/1-tumors, isoform M1-specific probeset indicated lower expression in stage 4+ tumor. (B) Normalized intensity (NI) values for probeset 3631984 in Stage 1-, 4- and 4+ tumors. (C) Normalized intensity values for probeset 3631977 in Stage 1-, 4- and 4+ tumors. The expression for probesets 3631984 and 3631977was significantly different between stage 1- and 4+, suggesting the increased expression of isoform M2 and reduced expression of isoform M1 in MYCN-amplified neuroblastoma.

Comparing stage 4- with 1 -, only 27 genes were shared between splicing signature $(n=362)$ and whole gene expression signature $(\mathrm{n}=185)$ (Figure $2 \mathrm{C})$. Using the Database for Annotation, Visualization and Integrated Discovery (DAVID) [22], we found that genes with known splice variants were significantly overrepresented in splicing signature $(n=153)$ but not expression signature based on UniProt annotation (FDR $<0.05$ ), which indicated the validity of our splicing analysis process. The most enriched Gene Ontology terms in the biological process category in the splicing signature included nervous system development, cell adhesion, synaptic transmission, and cytoskeleton organization and biogenesis (Figure 4A, Additional file 4). In contrast, the whole gene expression signature is enriched with genes involved in cell cycle, cell division, and DNA metabolic processes (Figure 4B, Additional file 4), which is consistent with the results derived using traditional gene expression microarray platforms [23]. Different sets of genes with different biological functions were affected by alternative splicing and transcription regulation, suggesting independent roles of splicing and transcription regulation in stage 4- neuroblastoma.

MYCN amplified neuroblastoma is the most aggressive disease subtype. By comparing stage 1 - and stage $4+$ neuroblastomas, we identified 9044 probesets within 2775 alternatively spliced candidate genes, along with 3298 differentially expressed genes between two tumor groups. Again, significant enrichment of genes with known splice variants $(\mathrm{n}=311)$ was found in those showing only splicing changes $(n=852)$, but not those having only whole gene expression changes ( $\mathrm{n}=$ 1375) $($ FDR < 0.05). In contrast to stage 4 - tumors, a large number of genes $(n=1923)$ appeared to have both changes in stage $4+$ tumors (Figure 2C). Biological processes enriched in alternatively spliced genes in stage 4+ include those involved in stage-specific splicing signature and whole gene expression signature (Table 2). Similar GO terms were enriched in genes showing whole gene expression changes in stage 4+ (Additional file 5). To derive $M Y C N$ amplification-specific signatures, we compared stage 4- and 4+ tumor, and detected 4602 probesets within 1501 genes that may undergo splicing disruption, and 2318 transcript clusters with expression changes during $M Y C N$ status change. The overlap between two signatures was also large with 1127 genes in common (Figure 2C). GO enrichment analysis results were similar to what was found in the comparison between stage $4+$ vs 1 tumors (Additional file 6). These results suggested a correlation between $M Y C N$ amplification and splicing regulation. While splicing and transcription regulation may affect different sets of genes involved in different biological processes in stage 4- tumors, these biological processes may be disrupted by both gene expression regulatory processes to a greater extent in the more severe stage 4+ neuroblastoma.

\section{Validation of splice variants differentially expressed in stage 1- and stage 4+ tumors}

Three alternatively spliced genes have been selected for quantitative RT-PCR validation including PKM2 (NM_002654 vs. NM_182470 \& NM_182471), KIF1B (NM_015074 vs. NM_183416) and MAP2 

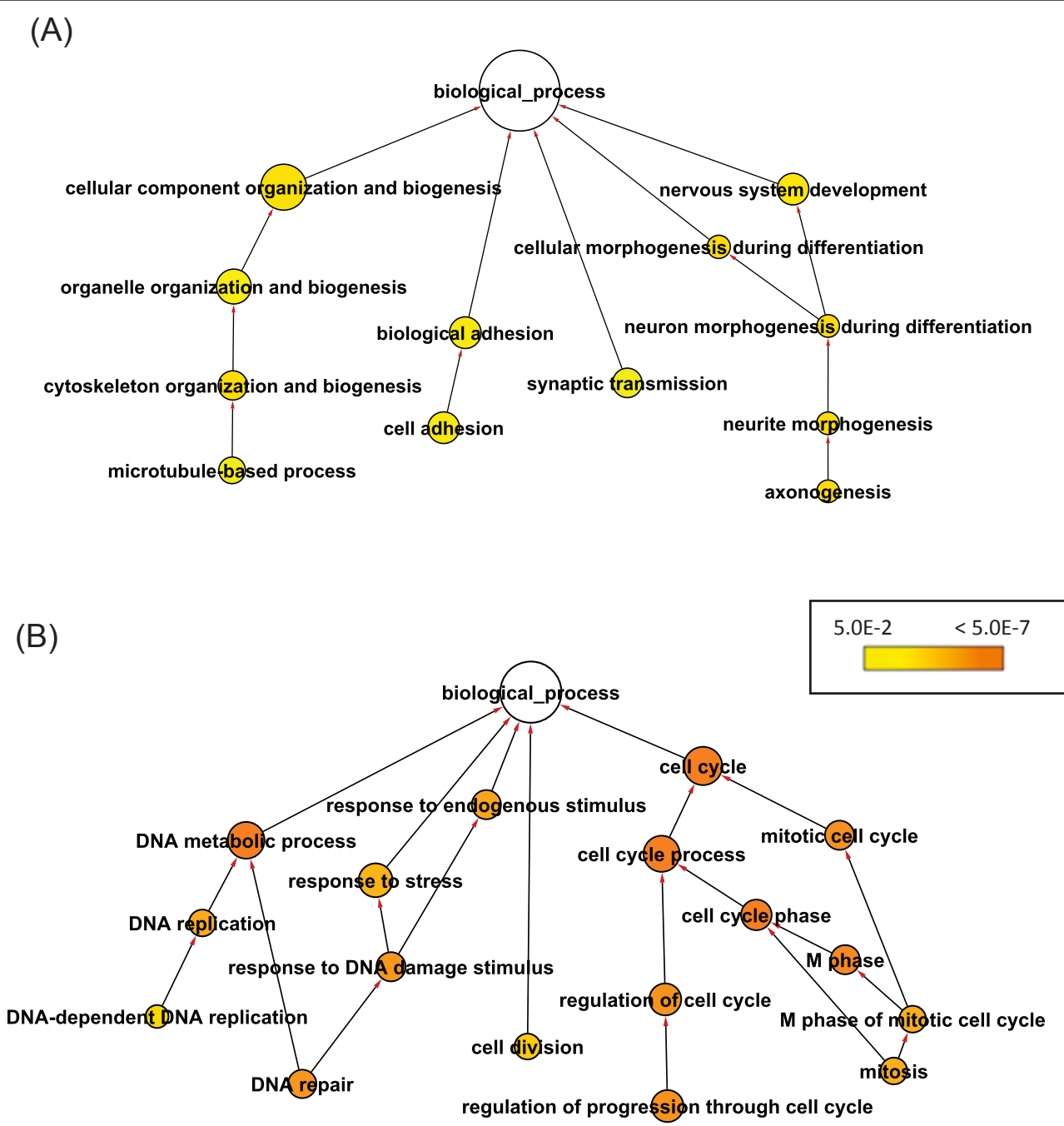

Figure 4 Enriched Gene Ontology (GO) biological processes in alternatively spliced (A) or differentially expressed (B) genes in stage 4vs 1- neuroblastomas. GO enrichment analysis was done by DAVID [22], and overrepresented biological processes were shown as a GO graph in which child terms are connected to their parent terms by directed lines. Color scale denotes Benjamini-corrected p-values generated by a hypergeometric test, and node size is proportional to the number of genes annotated to corresponding GO terms.

(NM_001039538 vs. NM_002374). Figure 5 shows gene expression fold changes between splice variants in 5 stage 1 - and 5 stage $4+$ tumors. The HuEx results for all three alternatively spliced genes were validated by qRTPCR result and the expression of splice variants is significantly different between stage 1 - and stage 4+ tumors with p-values (t-test) equal to 0.0038, 0.0014 and 0.0209 for genes PKM2, KIF1B and MAP2 respectively. The expression of M2 isoform (NM_002654) of PKM2 is increased in stage 4+ compared with M1 isoform; the expression of KIF1B $\beta$ (NM_015074) is decreased in stage $4+$ compared with KIF1B $\alpha$ (NM_002374); and the expression of isoform NM_001039538 of MAP2 is higher in stage 4+ compared with the isoform NM_002374.

\section{Discussion}

Alternative splicing of precursor mRNA is an essential step in gene expression and responsible for much of the proteome diversity in mammalian genomes. Although splice variants have long been known to be associated with many human diseases, very little is understood about the global properties of alternative splicing in cancer development. Using Affymetrix Human 1.0 exon array, we compared splicing interruption and whole gene expression change in different stages of a pediatric cancer - neuroblastoma. Our results suggested a significant role of splicing regulation in high stage and $M Y C N$ amplified neuroblastoma tumors.

In stage 4- neuroblastoma, transcription regulation and alternative splicing may affect different sets of genes 
Table 2 Top 40 overrepresented Gene Ontology biological processes (FDR $<0.0001$ ) in alternatively spliced genes in stage 4+ vs. 1- tumors

\begin{tabular}{|c|c|c|}
\hline GO Term & \# Gene & PValue \\
\hline cell cycle process ** & 203 & $2.26 \mathrm{E}-15$ \\
\hline mitotic cell cycle ** & 107 & $8.64 \mathrm{E}-15$ \\
\hline cellular component organization and biogenesis * & 572 & 1.07E-14 \\
\hline cell cycle ** & 230 & $1.33 \mathrm{E}-14$ \\
\hline cell cycle phase ** & 115 & $2.03 \mathrm{E}-14$ \\
\hline mitosis ** & 84 & $2.29 \mathrm{E}-14$ \\
\hline cell division ** & 88 & $3.86 \mathrm{E}-14$ \\
\hline M phase of mitotic cell cycle ${ }^{* *}$ & 84 & 4.03E-14 \\
\hline M phase ${ }^{* *}$ & 97 & $1.28 \mathrm{E}-13$ \\
\hline organelle organization and biogenesis * & 285 & 1.67E-13 \\
\hline developmental process & 651 & 4.37E-12 \\
\hline nervous system development ${ }^{*}$ & 192 & $1.66 \mathrm{E}-10$ \\
\hline multicellular organismal development & 479 & $4.24 \mathrm{E}-10$ \\
\hline cellular process & 2167 & $4.71 \mathrm{E}-10$ \\
\hline chromosome segregation & 32 & 7.47E-10 \\
\hline anatomical structure development & 441 & $1.43 \mathrm{E}-9$ \\
\hline localization & 610 & $1.10 \mathrm{E}-8$ \\
\hline regulation of cell cycle ** & 135 & $1.11 \mathrm{E}-8$ \\
\hline cell cycle checkpoint & 30 & $1.15 \mathrm{E}-8$ \\
\hline cytoskeleton organization and biogenesis * & 134 & $1.41 \mathrm{E}-8$ \\
\hline system development & 364 & $1.93 \mathrm{E}-8$ \\
\hline regulation of progression through cell cycle ** & 133 & $2.61 \mathrm{E}-8$ \\
\hline DNA replication ** & 74 & $7.81 \mathrm{E}-8$ \\
\hline synaptic transmission * & 84 & $2.63 \mathrm{E}-7$ \\
\hline transmission of nerve pulse & 93 & $2.98 \mathrm{E}-7$ \\
\hline establishment of localization & 533 & $5.36 \mathrm{E}-7$ \\
\hline intracellular signaling cascade & 307 & $6.77 \mathrm{E}-7$ \\
\hline cell proliferation & 179 & $7.05 \mathrm{E}-7$ \\
\hline transport & 516 & 7.30E-7 \\
\hline mitotic sister chromatid segregation & 18 & 7.33E-7 \\
\hline response to DNA damage stimulus ** & 86 & $1.18 \mathrm{E}-6$ \\
\hline sister chromatid segregation & 18 & $1.33 \mathrm{E}-6$ \\
\hline biological adhesion * & 173 & 1.67E-6 \\
\hline cell adhesion * & 173 & $1.67 \mathrm{E}-6$ \\
\hline DNA metabolic process ** & 188 & $2.59 \mathrm{E}-6$ \\
\hline cell communication & 747 & $7.35 \mathrm{E}-6$ \\
\hline interphase & 34 & $1.15 \mathrm{E}-5$ \\
\hline DNA replication initiation & 16 & $2.45 \mathrm{E}-5$ \\
\hline DNA-dependent DNA replication ** & 36 & $2.54 \mathrm{E}-5$ \\
\hline cell differentiation & 355 & $2.55 \mathrm{E}-5$ \\
\hline
\end{tabular}

* GO terms that are also enriched in alternatively spliced genes in stage 4- vs 1- tumors.

** GO terms that are also enriched in differentially expressed genes in stage 4- vs 1 - tumors.

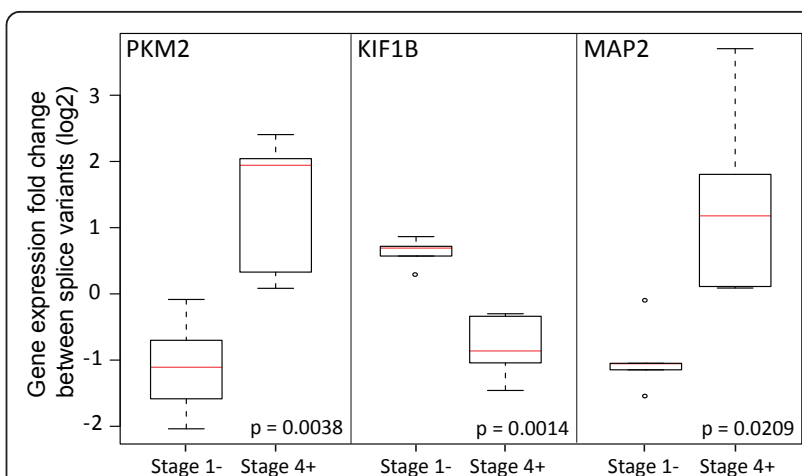

Figure 5 qRT-PCR validation on splice variants differentialy expressed in stage 1- and stage 4+ tumors. Quantitative RT-PCR was performed for three spliced genes including PKM2 (NM_002654 vs. NM 182470 \& NM_182471), KIF1B (NM_ 015074 vs. NM_183416) and MAP2 (NM_001039538 vs. NM_002374) in 5 stage 1- and 5 stage 4+ tumors. Differential expression of splice variants was evaluated by calculating expression fold changes between splice variants of the spliced gene in each sample, which were further centered by median of values obtained in stage 1- and stage 4+ tumors. The expression of splice variants is significantly different between stage 1- and stage 4+ tumors for all three tested spliced genes.

involved in different biological processes with more genes showing splicing disruption than whole gene expression change. Regulation of transcription and splicing seem to be two independent processes that result in distinct functional outcomes in stage 4- tumor, which is consistent with the independent roles of these two processes in determination of tissue specificity [24] and regulation of immune response [25]. In contrast, a large group of genes underwent both splicing and whole gene expression changes in stage $4+$ tumor. Similar biological processes were enriched in splicing and whole gene expression signatures in stage 4+ tumor, including those that were affected separately by alternative splicing and whole gene expression changes in stage 4- tumor. It indicates that different biological processes may be affected by splicing and transcription regulation in stage 4- tumors and these processes may need to be disrupted by both gene regulatory processes in the more severe stage 4+ tumors.

$M Y C N$-amplified tumor is a highly aggressive subtype with poor prognosis in $20 \%$ of neuroblastoma patients. Several studies have shown the differential expression of a large number of genes involved in cell cycle and differentiation in these tumors [23,26]. Our current study indicates, for the first time, that MYCN amplification is not only related to large scale gene expression changes but also profound splicing regulation in neuroblastoma. $M Y C N$ is a global transcriptional regulator for both 
protein-coding genes and genes encoding non-coding RNA products $[27,28]$. Transcriptional targets for $M Y C N$ include splicing factors [29] which may in turn regulate alternative splicing of various target genes. Recent study has shown that the splicing switch of pyruvate kinase in human gliomas may be controlled by cmyc through splicing factors including polypyrimidine tract binding protein (PTBP1) [30]. The PKM2 splicing switch (Figure 3) and PTBP1 expression upregulation we found in stage 4+ neuroblastoma (Additional file 3) suggest a $M Y C N$-controlled pathway for PKM2 splicing in neuroblastoma. Other splicing targets of PTBP1 have also been found in our splicing signatures of $M Y C N$ amplified tumors, including reticulon 4 (RTN4) [31] and ROD1 regulator of differentiation 1 [32]. In addition, multiple differentially expressed $M Y C / M Y C N$ target genes in stage 4+ tumor appear to be associated with splicing processing complex, such as small nuclear ribonucleoprotein polypeptides (SNRPA, SNRPB, SNRPD2), dead-box polypeptides (DDX1, DDX18), RNA-binding motif protein (RBM3), and cleavage and polyadenylation specific factor (CPSF1) [29,33]. These splicing regulators may be involved in $M Y C N$-associated splicing regulation, through which $M Y C N$ may exert part of its phenotypic effects on neuroblastoma.

Alternative splicing plays important roles in various diseases. It may be a direct cause of the disease, or a modifier of disease susceptibility and severity [2]. In this study, we identified candidate genes undergoing splicing disruption in high stage and MYCN amplified neuroblastoma, which help the understanding of disease biology. Neural development and cell adhesion genes exhibit splicing changes in stage 4- disease, and they undergo both splicing regulation and whole gene expression level change in stage $4+$ patients. Previous proteomics study has demonstrated that proteins involved in these processes are significantly suppressed in stage 4+ neuroblastoma patients [21]. Our results suggest that alternative splicing may be responsible, at least partially, for the changes observed at the proteome level. Defects in splicing machinery may cause alternative splicing and further whole gene expression changes of neural development and adhesion genes, resulting in the protein level changes observed in our previous study.

Alternatively spliced candidate genes identified in this study provides a useful resource for the discovery of diagnostic biomarkers or therapeutic targets in neuroblastoma. One example is related to splicing switch of PKM2 variants. Previous studies in multiple tumor types have shown that M2 isoform is expressed while M1 isoform disappears during tumor development [34]. PKM2 plays important role in cancer metabolism, and it has been proposed to be a potential metabolic target for the treatment of cancer $[19,35]$. Our results demonstrated the splicing switch of isoform M1 to isoform M2 in high stage neuroblastoma (Figure 3), suggesting a similar role of PKM2 in neuroblastoma development as in other tumors [35]. Another interesting result is related to the alternative splicing of microtubule associated genes. Our data demonstrated that genes involved in microtubuleassociated process were significantly enriched in differentially spliced candidate genes in stage 4+/4- vs stage 1 - tumors. There were 57, 27, and 15 microtubule-associated genes showing aberrant splicing pattern in stage 4 + vs 1-, 4+ vs 4-, and 4- vs 1- respectively (Figure 4A; Additional file 4, 5, 6). Among these genes, KIF1Bbeta but not alpha has been shown to be a potential 1 p36 tumor suppressor for neuroblastoma. Protein regulator of cytokinesis 1 (PRC1) is essential for organization of central spindle and midzone formation, whose interaction with KIF2C has been shown to be involved in breast cancer tumorigenesis [36]. Microtubule-associated protein 2 (MAP2) has been shown to be a prognostic marker for melanoma patients [37], and splice variants of MAPT gene demonstrated opposite changes in normal versus prostate tumor [38]. Recent studies have suggested a significant role of deregulated microtubule dynamics in enhanced genomic instabilities and tumor development [39]. Our results suggest that alteration of microtubule dynamics by alternative splicing may be an important pathogenetic mechanism in high stage neuroblastoma. Further functional study of these microtubuleassociated genes may reveal novel tumor suppressors or oncogenes for neuroblastoma.

\section{Conclusions}

In summary, our study demonstrated the important roles of splicing regulation in high-stage and $M Y C N$ amplified neuroblastoma. There may be a generalized shift in global splicing patterns synchronizing with the development of malignant phenotypes of tumor cells. Deciphering the "splicing code" is essential for our understanding of cancer etiology and progression pathway.

\section{Methods}

\section{Tumor samples}

We used 47 pretreatment primary neuroblastoma tumor samples in our study. All patients were blinded and anonymized to us. Our protocol was exempt from the NIH Multiple Project Assurance and our research activity involving human subjects was exempt from the office of Human Subjects Research (OHSR). The median age of forty-seven patients at diagnosis was 1.6 years (range 0.1 - 11.6 years). Ten patients had stage 1 disease while the remaining thirty-seven patients had stage 4 disease of which nine demonstrated MYCN gene amplification. 


\section{Array experiments and data analysis}

We extracted total RNA from tumor samples [40], which were then processed and labeled using Affymetrix whole transcript one-cycle labeling kit. After hybridization to Affymetrix Human Exon 1.0 arrays, chips were stained and scanned as per manufacturer's instructions. For data analysis, we used Affymetrix Power Tool (APT) to get signal intensities for probesets and transcript clusters. Probeset signals were estimated by PLIER and a detection p-value was assigned to each probeset by DABG algorithm. Gene-level signals were derived for 17800 transcript clusters comprised of core probesets that are supported by RefSeq transcripts and full-length mRNAs using IterPLIER algorithm. IterPLIER identifies highly correlated probesets of each transcript cluster to derive transcript cluster signal, thus gene-level expression estimate mostly includes the expression level of constitutive exons, which reflects gene expression regulation at the level of transcription and/or RNA stability but not splicing. A probeset is considered to be expressed in a sample if its detection p-value is less than 0.05 , and a transcript cluster is expressed if more than half of its consisting probesets have $\mathrm{p}$ values less than 0.05 .

For each core probeset $(\sim 284,000)$, we calculated normalized intensity (NI) which is probeset (exonlevel) intensity divided by transcript cluster (genelevel) intensity in each sample. The comparison of NI between two sample groups may reveal exons that are differentially spliced in two groups. To reduce the false positive rate in the splicing variant identification, several filtering steps were applied to the signals of both probesets and transcript clusters. First, the detection $p$-value was used to remove probesets and transcript clusters with undetectable signals. Probesets are required to be expressed in $>50 \%$ samples of at least one group, while transcript clusters have to be expressed in $>50 \%$ samples of both sample groups. Next, we removed probesets with very low variances based on the interquantile range of probeset intensities across all samples. The least variable $20 \%$ probesets were discarded to reduce the number of cross-hybridizing or non-responsive probesets. Thirdly, we set expression values of less than 30 to 30 to reduce noise [7]. Lastly, we reduced the effect of inaccurate annotations by removing transcript clusters with more than one annotated Entrez Gene identifiers as well as multiple transcript clusters annotated by the same Entrez Gene identifier.

After the filtering steps, we used the Significance Analysis of Microarray (SAM) [16] two class unpaired method to identify probesets that have statistically significant changes in NI values between two groups of patients. Briefly, a score $d_{i}$ is calculated for each probeset to measure the relative difference in splicing index between two groups of samples,

$$
d_{i}=\frac{\overline{N I}_{i 2}-\overline{N I}_{i 1}}{s_{i}+s_{0}}
$$

where $s_{i}$ is a pooled standard deviation over the two groups of samples, and $s_{o}$ is a small positive constant that adjusts for the small variability in the data. These $d_{i}$ values are used to rank probesets on ascending order and derive observed order statistics $d(i)$. Then, a permutation procedure $(n=100)$ is applied to get a set of permuted relative difference values and corresponding order statistics for each probeset. The average of permuted order statistics is defined as the expected statistic $d_{E}(i)$, which is plotted versus the observed statistic $d(i)$ in a scatter plot. The set of probesets that are away from the $d(i)=d E(i)$ line by a distance greater than an adjustable threshold $\Delta$ are called significant, and the percentage of such probesets identified by chance (false discovery rate) is estimated by the permuted dataset. In our study, the threshold $\Delta$ was chose to achieve a false discovery rate of 0.05 , and the fold change of NI was required to be more than 1.5. The analysis of SAM was done by the R package samr. Similarly, differentially expressed genes were identified by applying SAM on the signal estimate of transcript clusters. Non-specific filtering was applied to remove transcript clusters that are only expressed in less than $20 \%$ of all samples. The false discovery rate is also required to be less than 0.05 , and the signal fold change is more than 1.5.

For each probeset with significantly different NI between two tumor groups, we used AltAnalyze to identify competitive transcript isoforms that contain or do not contain the exon overlapping with that probeset. Possible splicing events caused by the alternative exon were classified into seven types (alternative N/C-terminus, alternative cassette exon, alternative 5 ' $/ 3$ ' splice site, retained intron, bleeding exon) based on the comparison of exon structures of competitive isoforms. Furthermore, we predicted changes in protein domains/motifs and miRNA binding sites associated with the probeset using both competitive isoform analysis and direct alignment method in AltAnalyze [17,41].

Gene-level signal was estimated by the IterPlier algorithm, and it mostly includes the expression level of constitutive exons, which reflects gene expression regulation at the level of transcription and/or RNA stability but not splicing.

\section{Array data access and visualization}

To facilitate exon array data access and visualization, we have developed a novel database and visualization system displaying both gene-level and exon-level 
expressions for array probesets and transcript clusters (http://pob.abcc.ncifcrf.gov/cgi-bin/JK). Coupled with exon array annotation tools, such as ArrayCheck [42], this system allows easy query and visual inspection of alternatively spliced exons. Clinical outcome was also integrated with gene expression data such that KaplanMaier plot may be dynamically generated for each probeset and transcript cluster. The raw data and the processed data are also available at http://www.ncbi.nlm. nih.gov/geo/ (GEO accession: GSE27608).

\section{Quantitative RT-PCR validation}

One microgram of total RNA was reversely transcribed to cDNA using Superscript II reverse transcriptase (Invitrogen) and random hexamer primer (Invitrogen) at $42^{\circ} \mathrm{C}$ for 1 hour. PCR reactions were performed with 40 cycles at $95^{\circ} \mathrm{C}$ for $30 \mathrm{sec}, 60^{\circ} \mathrm{C}$ for $30 \mathrm{sec}$, and $72^{\circ} \mathrm{C}$ for $45 \mathrm{sec}$ using SYBR Green PCR Master Mix (Applied Biosystems) and spicing variant-specific primers on ABI 7000 Sequence Detection System (Applied Biosystems). Splice variant-specific primers were designed using Primer 3 program (http://frodo.wi.mit.edu/primer3) and synthesized by Integrated DNA Technologies. Primer sequences are as follows: PKM2 (NM_182470\&NM_182471) forward 5'-CTA TCC TCT GGA GGC TGT GC-3', reverse 5'-GAG GCT CGC ACA AGT TCT TC-3'; PKM2 (NM_002654) forward 5'-ATC GTC CTC ACC AAG TCT GG-3', reverse 5'-GAA GAT GCC ACG GTA CAG GT-3'; KIF1B $\alpha$ (NM_183416) forward 5'- GAA GAT CGA AGA CGT CAT GGC C-3', reverse 5'-ACA CCA GCA CCA ACA GGC TCC-3'; KIF1B $\beta$ (NM_015074) forward 5'-ACT TCT AGC TGG CAC AAT ACG-3', reverse 5'-GTC ACC GTC AAG AAT CAC AAA G-3'; MAP2 (NM_002374) forward 5'-TGG GTG GAC ACT CAA GAT GA-3', reverse 5'-TGA TCT CCG AGC TTC CTT TT-3'; MAP2 (NM_001039538) forward 5'-GCT CTG GCT CCC AGT GTA TT-3', reverse 5'-CTG CTG AGG TGG GCT GTA TT-3'.

To evaluate differential expression of splice variants in stage 1- and stage 4+ tumors, we calculated expression fold changes between different splice variants of the spliced gene in each sample, which were further centered by median of values obtained in stage 1 - and stage $4+$ tumors.

\section{Additional material}

Additional file 1: Alternatively spliced candidate genes among stage 1-, 4-, and 4+ tumors detected by HuEx exon array study (FDR < 0.05).

Additional file 2: Alternative splicing of kinesin family member 1B (KIF1B) detected by Affymetrix exon array. While KIF1Balpha-specific probesets ( 6 probesets labeled by orange line) had no significant expression change, KIF1Bbeta-specific probesets (31 probesets to the right of the orange line) had significantly lower expression in high stage tumors.
Additional file 3: Candidate genes with whole gene expression changes among stage 1-, 4-, and 4+ tumors detected by HuEx exon array study (FDR $<0.05$ ).

Additional file 4: Gene Ontology enrichment of alternative spliced and/or differentially expressed candidate genes in stage 4- vs 1tumors.

Additional file 5: Gene Ontology enrichment of alternative spliced and/or differentially expressed candidate genes in stage 4+ vs 1tumors.

Additional file 6: Gene Ontology enrichment of alternative spliced and/or differentially expressed candidate genes in stage 4+ vs 4tumors.

\section{Acknowledgements}

This research was supported by the Intramural Research Program of the NIH, National Cancer Institute, Center for Cancer Research. We thank Drs. John Maris, Wendy London of the Children's Oncology Group (COG), Steven Qualman of the Cooperative Human Tissue Network (CHTN), Daniel Catchpoole at the Children's Hospital at Westmead, Australia, Frank Westermann, Manfred Schwab of German Cancer Research Center and Frank Berthold, University of Cologne, Germany for the tumor samples and patient demographic information. We thank Xinyu Wen and Jianbin He for their technical assistance in exon array database development. We also thank Drs. Shahab Asgharzadeh and Robert Seeger for the helpful discussions.

\section{Author details}

Oncogenomics Section, Pediatric Oncology Branch, National Cancer Institute, National Institute of Health, Gaithersburg, MD 20877, USA. ${ }^{2}$ Advanced Biomedical Computing Center, SAIC-Frederick, Inc., National Cancer Institute-Frederick, Frederick, MD 21702, USA.

\section{Authors' contributions}

$X G$ and QRC performed data analysis and wrote the manuscript. YKS carried out the experiments and helped interpret the data. JSW helped data analysis and revised the manuscript. JK conceived and supervised the study, and revised the manuscript. All authors read and approved the final manuscript.

\section{Competing interests}

The authors declare that they have no competing interests.

Received: 16 February 2010 Accepted: 18 April 2011

Published: 18 April 2011

\section{References}

1. Wang ET, Sandberg R, Luo S, Khrebtukova I, Zhang L, Mayr C, Kingsmore SF, Schroth GP, Burge CB: Alternative isoform regulation in human tissue transcriptomes. Nature 2008, 456(7221):470-476.

2. Wang GS, Cooper TA: Splicing in disease: disruption of the splicing code and the decoding machinery. Nat Rev Genet 2007, 8(10):749-761.

3. Thorsen K, Sorensen KD, Brems-Eskildsen AS, Modin C, Gaustadnes M, Hein AM, Kruhoffer M, Laurberg S, Borre M, Wang K, Brunak S, Krainer AR, Tørring N, Dyrskjøt L, Andersen CL, Orntoft TF: Alternative splicing in colon, bladder, and prostate cancer identified by exon-array analysis. Mol Cell Proteomics 2008.

4. Cheung HC, Baggerly KA, Tsavachidis S, Bachinski LL, Neubauer VL, Nixon TJ, Aldape KD, Cote GJ, Krahe R: Global analysis of aberrant premRNA splicing in glioblastoma using exon expression arrays. BMC Genomics 2008, 9(1):216.

5. Zhang C, Li HR, Fan JB, Wang-Rodriguez J, Downs T, Fu XD, Zhang MQ: Profiling alternatively spliced mRNA isoforms for prostate cancer classification. BMC Bioinformatics 2006, 7:202.

6. Klinck R, Bramard A, Inkel L, Dufresne-Martin G, Gervais-Bird J, Madden R, Paquet ER, Koh C, Venables JP, Prinos $P$, et al: Multiple alternative splicing markers for ovarian cancer. Cancer Res 2008, 68(3):657-663.

7. French PJ, Peeters J, Horsman S, Duijm E, Siccama I, van den Bent MJ, Luider TM, Kros JM, van der Spek P, Sillevis Smitt PA: Identification of 
differentially regulated splice variants and novel exons in glial brain tumors using exon expression arrays. Cancer Res 2007, 67(12):5635-5642.

8. Maris JM: Recent advances in neuroblastoma. N Engl J Med 362(23):2202-2211.

9. Brodeur GM, Pritchard J, Berthold F, Carlsen NL, Castel V, Castelberry RP, De Bernardi B, Evans AE, Favrot M, Hedborg F, et al: Revisions of the international criteria for neuroblastoma diagnosis, staging, and response to treatment. J Clin Oncol 1993, 11(8):1466-1477.

10. Oppenheimer O, Alaminos M, Gerald WL: Genomic medicine and neuroblastoma. Expert Rev Mol Diagn 2003, 3(1):39-54.

11. Seeger RC, Brodeur GM, Sather H, Dalton A, Siegel SE, Wong KY, Hammond D: Association of multiple copies of the N-myc oncogene with rapid progression of neuroblastomas. N Engl J Med 1985, 313(18):1111-1116

12. Tacconelli A, Farina AR, Cappabianca L, Desantis G, Tessitore A, Vetuschi A, Sferra R, Rucci N, Argenti B, Screpanti I, et al: TrkA alternative splicing: a regulated tumor-promoting switch in human neuroblastoma. Cancer Cell 2004, 6(4):347-360

13. Munirajan AK, Ando K, Mukai A, Takahashi M, Suenaga Y, Ohira M, Koda T, Hirota T, Ozaki T, Nakagawara A: KIF1Bbeta functions as a haploinsufficient tumor suppressor gene mapped to chromosome 1 p36.2 by inducing apoptotic cell death. J Biol Chem 2008, 283(36):24426-24434

14. Schlisio S, Kenchappa RS, Vredeveld LC, George RE, Stewart R, Greulich H, Shahriari K, Nguyen NV, Pigny P, Dahia PL, et al: The kinesin KIF1Bbeta acts downstream from EgIN3 to induce apoptosis and is a potential 1 p36 tumor suppressor. Genes Dev 2008, 22(7):884-893.

15. Srinivasan K, Shiue L, Hayes JD, Centers R, Fitzwater S, Loewen R, Edmondson LR, Bryant J, Smith M, Rommelfanger $C$, et al: Detection and measurement of alternative splicing using splicing-sensitive microarrays. Methods 2005, 37(4):345-359.

16. Tusher VG, Tibshirani R, Chu G: Significance analysis of microarrays applied to the ionizing radiation response. Proc Natl Acad Sci USA 2001, 98(9):5116-5121.

17. Emig D, Salomonis N, Baumbach J, Lengauer T, Conklin BR, Albrecht M: AltAnalyze and DomainGraph: analyzing and visualizing exon expression data. Nucleic Acids Res 38(Suppl):W755-762.

18. Chen YY, Takita J, Chen YZ, Yang HW, Hanada R, Yamamoto K, Hayashi Y: Genomic structure and mutational analysis of the human KIF1Balpha gene located at 1p36.2 in neuroblastoma. Int J Oncol 2003, 23(3):737-744.

19. Christofk HR, Vander Heiden MG, Harris MH, Ramanathan A, Gerszten RE, Wei R, Fleming MD, Schreiber SL, Cantley LC: The M2 splice isoform of pyruvate kinase is important for cancer metabolism and tumour growth. Nature 2008, 452(7184):230-233.

20. Dombrauckas JD, Santarsiero BD, Mesecar AD: Structural basis for tumor pyruvate kinase M2 allosteric regulation and catalysis. Biochemistry 2005, 44(27):9417-9429.

21. Chen QR, Song YK, Yu LR, Wei JS, Chung JY, Hewitt SM, Veenstra TD, Khan J: Global genomic and proteomic analysis identifies biological pathways related to high-risk neuroblastoma. J Proteome Res 2010, 9(1):373-382

22. Huang da W, Sherman BT, Lempicki RA: Systematic and integrative analysis of large gene lists using DAVID bioinformatics resources. Nat Protoc 2009, 4(1):44-57.

23. Krasnoselsky AL, Whiteford CC, Wei JS, Bilke S, Westermann F, Chen QR, Khan J: Altered expression of cell cycle genes distinguishes aggressive neuroblastoma. Oncogene 2005, 24(9):1533-1541.

24. Pan Q, Shai O, Misquitta C, Zhang W, Saltzman AL, Mohammad N, Babak T, Siu H, Hughes TR, Morris QD, et al: Revealing global regulatory features of mammalian alternative splicing using a quantitative microarray platform. Mol Cell 2004, 16(6):929-941.

25. Ip JY, Tong A, Pan Q, Topp JD, Blencowe BJ, Lynch KW: Global analysis of alternative splicing during T-cell activation. RNA 2007, 13(4):563-572.

26. Alaminos M, Mora J, Cheung NK, Smith A, Qin J, Chen L, Gerald WL: Genome-wide analysis of gene expression associated with MYCN in human neuroblastoma. Cancer Res 2003, 63(15):4538-4546.

27. Westermann F, Muth D, Benner A, Bauer T, Henrich KO, Oberthuer A, Brors B, Beissbarth T, Vandesompele J, Pattyn F, et al: Distinct transcriptional MYCN/c-MYC activities are associated with spontaneous regression or malignant progression in neuroblastomas. Genome Biol 2008, 9(10):R150
28. Meyer N, Penn LZ: Reflecting on 25 years with MYC. Nat Rev Cancer 2008, 8(12):976-990

29. Zeller KI, Jegga AG, Aronow BJ, O'Donnell KA, Dang CV: An integrated database of genes responsive to the Myc oncogenic transcription factor: identification of direct genomic targets. Genome Biol 2003, 4(10):R69.

30. David CJ, Chen M, Assanah M, Canoll P, Manley JL: HnRNP proteins controlled by c-Myc deregulate pyruvate kinase mRNA splicing in cancer. Nature 463(7279):364-368

31. Cheung HC, Hai T, Zhu W, Baggerly KA, Tsavachidis S, Krahe R, Cote GJ: Splicing factors PTBP1 and PTBP2 promote proliferation and migration of glioma cell lines. Brain 2009, 132(Pt 8):2277-2288

32. Spellman R, Llorian M, Smith CW: Crossregulation and functional redundancy between the splicing regulator PTB and its paralogs $\mathrm{NPTB}$ and ROD1. Mol Cell 2007, 27(3):420-434.

33. Chen YI, Moore RE, Ge HY, Young MK, Lee TD, Stevens SW: Proteomic analysis of in vivo-assembled pre-mRNA splicing complexes expands the catalog of participating factors. Nucleic Acids Res 2007, 35(12):3928-3944.

34. Mazurek S, Boschek CB, Hugo F, Eigenbrodt E: Pyruvate kinase type M2 and its role in tumor growth and spreading. Semin Cancer Biol 2005, 15(4):300-308.

35. Kroemer G, Pouyssegur J: Tumor cell metabolism: cancer's Achilles' heel. Cancer Cell 2008, 13(6):472-482.

36. Shimo A, Nishidate T, Ohta T, Fukuda M, Nakamura Y, Katagiri T: Elevated expression of protein regulator of cytokinesis 1 , involved in the growth of breast cancer cells. Cancer Sci 2007, 98(2):174-181.

37. Soltani MH, Pichardo R, Song Z, Sangha N, Camacho F, Satyamoorthy K, Sangueza OP, Setaluri V: Microtubule-associated protein 2, a marker of neuronal differentiation, induces mitotic defects, inhibits growth of melanoma cells, and predicts metastatic potential of cutaneous melanoma. Am J Pathol 2005, 166(6):1841-1850

38. Li HR, Wang-Rodriguez J, Nair TM, Yeakley JM, Kwon YS, Bibikova M, Zheng C, Zhou L, Zhang K, Downs T, et al: Two-dimensional transcriptome profiling: identification of messenger RNA isoform signatures in prostate cancer from archived paraffin-embedded cancer specimens. Cancer Res 2006, 66(8):4079-4088.

39. Rao CV, Yamada HY, Yao Y, Dai W: Enhanced genomic instabilities caused by deregulated microtubule dynamics and chromosome segregation: a perspective from genetic studies in mice. Carcinogenesis 2009, 30(9):1469-1474

40. Wei JSKJ: Purification of total RNA from mammalian cells and tissues. In DNA microarrays: a molecular cloning manual. Edited by: D Bowtell JS. Cold Spring Harbor, New York: Cold Spring Harbor Laboratory Press; 2002:110-119.

41. Salomonis N, Nelson B, Vranizan K, Pico AR, Hanspers K, Kuchinsky A, Ta L, Mercola M, Conklin BR: Alternative splicing in the differentiation of human embryonic stem cells into cardiac precursors. PLoS Comput Biol 2009, 5(11):e1000553.

42. Ryan MC, Zeeberg BR, Caplen NJ, Cleland JA, Kahn AB, Liu H, Weinstein JN: SpliceCenter: a suite of web-based bioinformatic applications for evaluating the impact of alternative splicing on RT-PCR, RNAi, microarray, and peptide-based studies. BMC Bioinformatics 2008, 9:313.

\section{Pre-publication history}

The pre-publication history for this paper can be accessed here: http://www.biomedcentral.com/1755-8794/4/35/prepub

doi:10.1186/1755-8794-4-35

Cite this article as: Guo et al:: Exon array analysis reveals neuroblastoma tumors have distinct alternative splicing patterns according to stage and MYCN amplification status. BMC Medical Genomics 2011 4:35. 